Another important point relates to any assumptions that trials published before the 1996 CONSORT statement are more likely to be of poorer quality than those published after it. ${ }^{3}$ The CONSORT statement contains several methodological elements that should be followed to eliminate biased results. The intention of this statement was to improve the conduct, integrity, and reporting of randomised controlled trials. ${ }^{3}$ Our results show that studies conducted by the Radiation Therapy Oncology Group were of high quality even before publication of the CONSORT statement. It was the reports of the randomised controlled trials that showed deficiencies in their description of the methods used in the trials, not the trials themselves. Our findings indicate that although researchers in the Radiation Therapy Oncology Group were cognisant of key features in the design and conduct of good quality trials, they were less aware of the need to report these to a standard that would meet contemporary (CONSORT) requirements.

It is still appropriate to expect that the CONSORT statement will contribute to the conduct of higher quality randomised controlled trials in the future, since it incorporates and highlights many of the elements needed to perform a trial adequately. We agree with the call for all journals to adopt the policy of only publishing the report of a randomised controlled trial if it follows the CONSORT requirements. This is supported by empirical data that are now emerging about the usefulness of the CONSORT statement. For example, one study compared the quality of reports of trials before and after the CONSORT statement and found that the statement was associated with an improvement in the quality of reports. ${ }^{12}$ Further improvements in the quality of the conduct and reporting of clinical research would arise with the publication of research protocols.

Contributors: HPS and BD conceptualised the study, were involved in all aspects of the study, and wrote the first draft of the paper. SD and AK contributed to the study design, collection of data, analysis and interpretation of the data, and writing the report. MC contributed to the study design, interpretation of the data, and writing the report. CS and SS contributed to the collection of data and writing the report. BD will act as guarantor for the paper.

Funding: This research was supported by the Research Program on Research Integrity, an Office of Research Integrity/National Institute of Health collaboration, grant No 1R01NS/NR44417-01.

\section{What is already known on this topic}

Assessment of the quality of research evidence is central to informed decision making

The quality of randomised controlled trials is often based on the quality of reporting

What this study adds

Poor reporting of methods in randomised controlled trials may not reflect on poor methods themselves

Evaluation of research protocols and contacting trialists should be integral to assessing the quality of such trials

Competing interests: MC is director of the UK Cochrane Centre, which is funded by the NHS research and development programme and part of the international Cochrane Collaboration. The collaboration produces systematic reviews of health care, including randomised trials, but the views expressed here are not necessarily those of the official policy of the Cochrane Collaboration.

Ethical approval: This study was approved by the University of South Florida Institutional Review Board (No 100449).

1 Moher D, Pham B, Jones A, Cook DJ, Jadad AR, Moher M, et al. Does quality of reports of randomised trials affect estimates of intervention efficacy reported in meta-analyses? Lancet 1998;352:609-13.

2 Huwiler-Muntener K, Juni P, Junker C, Egger M. Quality of reporting of randomized trials as a measure of methodologic quality. JAMA 2002;287:2801-4

3 Moher D, Schulz KF, Altman D. The CONSORT statement: revised recommendations for improving the quality of reports of parallel-group randomized trials. JAMA 2001;285:1987-91.

4 Godlee F. Publishing study protocols: making them visible will improve registration, reporting and recruitment. BMC News Views 2001;2:4

5 Silagy CA, Middleton P. Hopewell S. Publishing protocols of systematic reviews: comparing what was done to what was planned. JAMA reviews: comparing

2002;287:2831-4. RTOG procedure manual. Philade

7 Egger M, Davey Smith G, Altman D. Systematic reviews in health care. Meta analysis in context, 2nd ed. London: BMJ Publishing, 2001.

Clarke M, Oxman A, eds. Cochrane reviewers' handbook 4.2.0 [updated March 2003]. In Cochrane Library, issue 2. Oxford: Update Software, 2003.

Liberati A, Himel HN, Chalmers TC. A quality assessment of randomized control trials of primary treatment of breast cancer. J Clin Oncol 1986; 4:942-51.

10 Herson J. Patient registration in a cooperative oncology group. Control Clin Trials 1980;1:101-10.

11 Schulz KF, Chalmers I, Hayes RJ, Altman DG. Empirical evidence of bias. Dimensions of methodological quality associated with estimates of treatment effects in controlled trials. JAMA 1995;273:408-12.

12 Moher D, Jones A, Lepage L. Use of the CONSORT statement and quality of reports of randomized trials: a comparative before-and-after evaluation. JAMA 2001;285:1992-5.

(Accepted 10 October 2003)

\title{
Commentary: The quality of randomised controlled trials may be better than assumed
}

Auro del Giglio, Luciano Jose Costa

ABC Foundation School of Medicine, Avenida Reboucas 3387 São Paulo, Brazil, 05410-040 Auro del Giglio chairman of haematology and oncology continued over
As readers of published articles, it is reassuring to know that the quality of published data is probably better than expected from the reporting of the methods. Soares and colleagues have addressed the discrepancies between the proposed methods in original research protocols and those reported in the final article for all 56 randomised controlled trials conducted by the Radiation Therapy Oncology Group since its creation in 1968. Good quality experimental designs were more often adhered to during the conduct of the studies than suggested by the final reports.

As readers of systematic reviews, however, we may have underestimated the quality of experimental methods, especially if reviewers had not checked the original protocols. This may have led to the exclusion of some potentially good quality papers from systematic 
reviews. ${ }^{1}$ This would also affect the scoring of quality of papers as a part of a meta-analysis. ${ }^{23}$ Soares and colleagues suggest that this problem can be avoided by accessing the original protocols and contacting trialists to check the accuracy of reported methods. Authors should also adhere to the Consolidated Standards of Reporting of Trials (CONSORT) statement. ${ }^{4}$

In the near future, papers may be accompanied by internet links to their original protocol and data, allowing researchers to download crude data for metaanalysis. Until that time, it should be borne in mind that although papers fail to report important design and methodological features, they were probably done, especially if the papers are authored by a body such as the Radiation Therapy Oncology Group, the protocols of which undergo rigorous review.
Contributors: AdG was the main author of the commentary and wrote the final manuscript. He will act as guarantor. LJC made modifications to the manuscript and contributed to the final manuscript.

Funding: None.

Competing interests: None declared.

1 Pignon JP, Hill C. Meta-analyses of randomised clinical trials in oncology. Lancet Oncol 2001;2:475-82.

2 Detsky AS, Naylor CD, O'Rourke K, McGeer AJ, L'Abbe KA. Incorporating variations in the quality of individual randomized trials into meta-analysis. J Clin Epidemiol 1992:45:255-65.

3 Moher D, Pham B, Jones A, Cook DJ, Jadad AR, Moher M, et al. Does quality of reports of randomised trials affect estimates of intervention efficacy reported in meta-analyses? Lancet 1998;352:609-13.

4 Moher D, Schulz KF, Altman DG. The CONSORT statement: revised recommendations for improving the quality of reports of parallel-group randomised trials. Lancet 2001:357:1191-4.
ABC Foundation

School of Medicine

Division of Medical

Oncology,

University of

Colorado Health

Sciences Center,

Denver, CO 80262

USA

Luciano Jose Costa

clinical fellow in

haematology and

medical oncology

Correspondence to:

A del Giglio

sandrabr@

netpoint.com.b

\section{Drug points}

\section{Rash and acute nephritic syndrome due to candesartan \\ Adam Morton, James Muir, Davin Lim}

A 73 year old man presented with a two day history of a pruritic rash and oedema affecting both lower legs. He had had hypertension for three years, for which he had been taking candesartan, an angiotensin II receptor antagonist, for the past two years. He was not taking any other medications or preparations. He had no infective, gastrointestinal, or respiratory symptoms and no arthralgias.

Examination showed non-palpable, non-tender purpura affecting both legs, with pitting oedema, and urticarial lesions on the left knee and anterior chest. Testing of urine was positive for protein and blood. Urine microscopy showed $>100$ erythrocytes per $10^{6} / \mathrm{l}$; the urine protein:creatinine ratio was 0.15 (normal range $<0.04)$, serum creatinine was $90 \mu \mathrm{mol} / \mathrm{l}(70-120 \mu \mathrm{mol} / \mathrm{l})$, $\mathrm{C}$ reactive protein was $117 \mathrm{mg} / \mathrm{l}(0-6 \mathrm{mg} / \mathrm{l})$, and the erythrocyte sedimentation rate was $32 \mathrm{~mm} /$ hour (1-20 $\mathrm{mm}$ /hour). A provisional diagnosis of Schönlein-Henoch purpura due to candesartan was made, and the medication was stopped. Serum immunoglobulin A concentration, however, was normal. The results of an autoantibody screen and testing for antineutrophil cytoplasmic antibodies were negative, complements were normal, and cryoglobulins were not detected. A skin biopsy showed lymphocytic vasculitis involving vessels in the papillary and mid-dermis, as well as spongiosis and moderate orthokeratosis. We believed the features to be consistent with a drug reaction. The rash and microscopic haematuria resolved completely within a week of stopping candesartan and $\mathrm{C}$ reactive protein concentration became normal three weeks after presentation, though proteinuria took 10 weeks to resolve.

Irbesartan, another angiotensin II receptor antagonist, was the highest volume drug prescribed for hypertension in Australia on the pharmaceutical benefits scheme for the year ending December 2002. A major reason for the popularity of this class of drugs is their side effect profile, shown to be similar to that of placebo in double blind studies. ${ }^{1}$ Major side effects published in the literature, however, include hepatotoxicity, pancreatitis, angio-oedema, acute deterioration in renal function, and dysgeusia. Two cases of Schönlein-Henoch purpura have been described as being associated with therapy with losartan. ${ }^{23}$ Both had a similar presentation to the case described above, with purpuric rash, pedal oedema, microscopic haematuria, proteinuria, raised $\mathrm{C}$ reactive protein concentration, and rapid resolution when the drug was stopped. Both cases, however, were associated with raised serum IgA concentration and deposits in the dermal vessel walls on histology.

From 1999 until November 2002, seven cases of rash were reported to the Adverse Drug Reaction Advisory Committee in Australia, in which candesartan was the sole possible agent responsible. No cases of nephritis have been reported to the committee to date. Similarly, the manufacturer of candesartan (AstraZeneca) has not received any reported cases of nephritis with candesartan. In conclusion, the angiotensin II receptor antagonists are a very well tolerated group of antihypertensive drugs, but they should be considered to be a potential cause of rash or acute nephritic syndrome in any patients presenting with those symptoms, regardless of how long they have been taking the drug.

Funding: None.

Competing interests: None declared.

1 Andersson OK Tolerability of a modern antihypertensive agent: candesartan cilexetil. Basic Res Cardiol 1998;939(suppl 2):54-8.

Bosch X. Henoch-Schonlein purpura induced by losartan therapy. Arch Bosch X. Henoch-Schonlei
Intern Med 1998;158:191-2.

Intern Med 1998;158:191-2.
Brouard M, Piguet V, Chavaz P, Borradori L. Schonlein-Henoch purpura associated with losartan treatment and presence of antineutrophil cytoplasmic antibodies of x specificity. Br J Dermatol 2001;145:362-3.

\section{Endpiece}

\section{Students at Guy's and}

\section{St Thomas's, 1819}

I must be permitted to caution you against blindly adopting any system of opinion and practice which may be taught in the schools to which you may be ... attached; this could be to degrade you to the ranks of empiricism. Think for yourselves.

Aesculapius. The hospital pupil's guide at St Thomas's and Guy's Hospitals. Lond Med Repository 1819;11:128-30

Jeremy Hugh Baron,

honorary professorial lecturer,

Mount Sinai School of Medicine, New York
Mater Adult

Hospital, South Brisbane, Brisbane, Australia, 4101

Adam Morton

physician

James Muir

visiting dermatologist

Davin Lim

dermatology registrar Correspondence to: Adam Morton amorton@ mater.org.au

BMJ 2004;328:25 